\title{
Relativistic dynamics of cylindrical shells of counter-rotating particles
}

\author{
V. H. Hamity*, M. A. Cécere †and D.E. Barraco \\ Fa.M.A.F., Universidad Nacional de Córdoba \\ Ciudad Universitaria, Córdoba 5000, Argentina
}

August 25, 2021

\begin{abstract}
Although infinite cylinders are not astrophysical entities, it is possible to learn a great deal about the basic qualitative features of generation of gravitational waves and the behavior of the matter conforming such shells in the limits of very small radius. We describe the analytical model using kinetic theory for the matter and the junction conditions through the shell to obtain its equation of motion. The nature of the static solutions are analyzed, both for a single shell as well as for two concentric shells. In this second case, for a time dependent external shell, we integrate numerically the equation of motion for several values of the constants of the system. Also, a brief description in terms of the Komar mass is given to account for the gravitational wave energy emitted by the system.
\end{abstract}

\section{Introduction}

Recently there has been a growing interest in the literature to study the gravitational dynamics of infinite cylindrical shells of counter-rotating particles in different geometrical settings[1]. One of the best studied case is that considered

\footnotetext{
*hamity@famaf.unc.edu.ar

$\dagger$ cm4@famaf. unc . edu. ar

†barraco@famaf.unc.edu.ar
} 
by Apostolatos and Thorne[2]. They made a detailed analysis of the system using C-energy balance arguments[3] and a sequence of momentarily static and radiation-free shells to avoid the complications causes by gravitational radiation for a fully relativistic cylindrical shell. However, for a shell outside equilibrium the radiation-free requirement is incompatible with the shell equation of motion, obtained from the matching conditions of the interior and exterior vacuum solutions. In this paper we show that there are two equilibrium solutions, corresponding to constants values of shell radius, the rest mass per unit of proper length $(\Lambda)$ and the angular momentum per unit of rest mass $(J)$ of the particles composing the shell. One of the solution is a stable equilibrium while the other is unstable. Next, we study a system of two shells, first in a static configuration and show that the equilibrium configuration of the external shell may also be stable or unstable in a similar way as for only one shell; then, we integrate (numerically) the equation of motion of the external shell for different values of the constants of motion and initial values of the coordinate radius starting at rest. This integration is possible, without having a complete knowledge of the wave solution outside the external shell, because the matching conditions and the fact that the internal solutions satisfies the time independent field equations. The innermost shell is chosen in a static stable configuration, which allows us to have a smooth spacetime geometry on the cylinder's symmetry axis. We obtain shells that perform damped oscillations, collapse or are locally expanding, depending on the values of the conserved quantities and integration constants. We give a brief description of the energy emitted by the system in the case of damped oscillations, in terms of a generalized Komar mass.

The paper is organized as follows. In the next section we describe the geometry and equation of motion of a cylindrical shell of counter-rotating particles. This includes the metric and junction conditions and the description of the surface stress-energy tensor using the kinetic theory in general Relativity adapted to the shell. In section 3 we discuss the stable and unstable equilibrium solutions of a single shell. In section 4 the dynamics of two concentric cylindrical shells of counter-rotating particles is presented and the matter equation of motion is numerically integrated to show the different types of solutions, depending on the values of the conserved quantities and integration constants. Finally, a brief summary of the main results is given in section 5 . 


\section{Geometry and equation of motion of a cylindrical shell of counter-rotating particles}

\subsection{Metric and junction conditions}

We consider a spacetime $M=M^{-} \cup \Sigma \cup M^{+}$with cylindrical symmetry where $\Sigma$ is the history of a hollow cylinder composed of counter-rotating particles of rest mass equal to unity; $M^{-}\left(M^{+}\right)$is the vacuum interior (exterior) region of the cylinder. In the vacuum interior $\left(M^{-}\right)$and exterior $\left(M^{+}\right)$of the shell, we introduce canonical cylindrical coordinates $(t, r, z, \phi)$. The metric takes the form[3].

$$
d s_{ \pm}^{2}=e^{2 \gamma_{ \pm}-2 \psi_{ \pm}}\left(d r^{2}-d t_{ \pm}^{2}\right)+e^{2 \psi_{ \pm}} d z^{2}+e^{-2 \psi_{ \pm}} r^{2} d \phi^{2}
$$

These coordinates are uniquely determined up to the (non-trivial) change of scale

$$
z \longrightarrow e^{\mu} z, \quad \psi \longrightarrow \psi-\mu, \quad r \longrightarrow e^{-\mu} r, \quad t \longrightarrow e^{-\mu} t .
$$

The Einstein field equations in the empty space inside and outside the shell are

$$
\begin{gathered}
\psi_{, r r}+\frac{1}{r} \psi_{, r}-\psi_{, t t}=0 \\
\gamma_{, t}=2 r \psi_{, r} \psi_{, t}, \quad \gamma_{, r}=r\left(\psi_{, r}^{2}+\psi_{, t}^{2}\right) .
\end{gathered}
$$

Thus, $\psi(r, t)$ plays the role of a gravitational field whose static part is analogue of the Newtonian potential. The time depended solutions of (3) represent gravitational waves [4]. Equation (3) is the integrability condition of equations (4). The coordinates $(z, \phi, r)$ and the metric function $\psi$ are continuous across the shell $\Sigma$, while $t$ and the metric function $\gamma$ are discontinuous. Smoothness of the spacetime geometry on the axis $r=0$ requires that

$$
\gamma=0 \text { and } \psi \text { finite at } r=0
$$

The junction conditions of $M^{-}$and $M^{+}$through $\Sigma$ require the continuity of the metric and to specify the jump of the extrinsic curvature $K^{ \pm}$compatible with the stress-energy tensor on the shell. The induced metric on $\Sigma$ is given by

$$
d s_{\Sigma}^{2}=-d \tau^{2}+e^{2 \psi_{\Sigma}} d z^{2}+e^{-2 \psi_{\Sigma}} R^{2} d \phi^{2}
$$

Here $\psi_{\Sigma}(\tau)=\psi_{+}\left(R(\tau), t_{+}(\tau)\right)=\psi_{-}\left(R(\tau), t_{-}(\tau)\right)$; the evolution of the shell is characterized by $R(\tau)$, which is the radial coordinate $r$ at the shell's location and 
$\tau$ the proper time of an observer at rest on $\Sigma$. The Einstein field equations on the shell reduce to[5]

$$
K_{i j}^{+}-K_{i j}^{-}=8 \pi\left(S_{i j}-\frac{1}{2} S g_{i j}\right) \text {; }
$$

here $S_{i j}$ es the surface stress-energy tensor, $g_{i j}$ is given by (6) $[i=(\tau, z, \phi)]$ and

$$
K_{i j}^{ \pm}=-\left(h_{d}^{c} h_{b}^{a} N_{c ; a}^{ \pm}\right) e_{i}^{d} e_{j}^{b} ; \quad e_{i}=\left(\frac{\partial}{\partial \tau}, \frac{\partial}{\partial z}, \frac{\partial}{\partial \phi}\right) ; \quad h_{d}^{c}=\delta_{d}^{c}-N_{d} N^{c},
$$

$N$ is the outward unit vector normal to the shell:

$$
N=N^{r} \frac{\partial}{\partial r}+N^{t} \frac{\partial}{\partial t} ; \quad N^{r}>0 \text {. }
$$

The tangent vector is

$$
\frac{\partial}{\partial \tau}=\dot{R} \frac{\partial}{\partial r}+X^{ \pm} \frac{\partial}{\partial t^{ \pm}}
$$

From the normalization conditions $(u \cdot u=-1, N \cdot N=1)$ we get

$$
\frac{\partial t_{ \pm}}{\partial \tau} \equiv X^{ \pm}=+\sqrt{e^{-2\left(\gamma_{ \pm}-\psi_{\Sigma}\right)}+\dot{R}^{2}}
$$

Similarly, from $u \cdot N=0$, we get

$$
N^{r}=X^{ \pm}, \quad N^{t}=\dot{R} .
$$

The calculation of the tensors $K_{i j}^{ \pm}$gives the result (we omit the ( \pm ) for simplicity)

$$
\begin{aligned}
K_{\tau \tau} & =\left(\gamma_{, t}-\psi_{, t}\right) \dot{R}+X\left(\gamma_{, r}-\psi_{, r}\right)-(\dot{R} \dot{X}-\ddot{R} X) e^{2 \gamma-2 \psi_{\Sigma}}, \\
K_{\phi \phi} & =-R^{2} e^{-2 \psi_{\Sigma}}\left[\left(\frac{1}{R}-\psi_{, r}\right) X-\psi_{, t} \dot{R}\right], \\
K_{z z} & =-e^{2 \psi_{\Sigma}}\left[\psi_{, r} X+\psi_{, t} \dot{R}\right] .
\end{aligned}
$$

All the quantities are computed at the location of the shell $\Sigma$.

\subsection{The surface stress-energy tensor}

To compute the surface stress-energy tensor we shall use the relativistic kinetic theory [6] adapted to the matter within the shell $\Sigma$.

The stress-energy tensor at the event $x \in \Sigma$ is defined in the usual way by

$$
\begin{aligned}
T^{a b}(x) & =\int_{P(x)} p^{a} p^{b} f(x, p) \pi \\
& =S^{a b} \delta(n),
\end{aligned}
$$


here $f(x, p)$ is the distribution function; $P(x)$ is the space of momentum $p=$ $\left(p^{a} \partial / \partial x^{a}\right)$ in $x$, corresponding to particles with proper mass $m=1, p \cdot p=-1$, and $\pi$ is the intrinsic element of volume in this space. Introducing a coordinate $n$ normal to $\Sigma$, such that $n=0$ defines $\Sigma$, we have $x^{a}=\left(n, x^{i}\right)$ and

$$
\begin{gathered}
S^{a n}=S^{n a}=0, \\
S^{i j}=\int_{\tilde{P}(x)} p^{i} p^{j} \tilde{f} \tilde{\pi}, \\
\tilde{\pi}=\frac{\sqrt{-g}}{\left|p_{\tau}\right|} d p^{z} d p^{\phi}, \quad g=\operatorname{det}\left(g_{i j}\right) .
\end{gathered}
$$

Here $\tilde{P}(x)=\{p: p \in P(x), p \cdot n=0\} ; \tilde{f}$ is the restriction to $\Sigma$ of the distribution function $f$. In absence of collisions, $\tilde{f}$ satisfies the Liouville equation in the oneparticle phase space $\tilde{M}=\{(x, p) / x \in \Sigma ; p \in \tilde{P}(x)\}$.

$$
\tilde{L}(\tilde{f}) \equiv \frac{d \tilde{f}}{d \tau}=0
$$

where $(d \tilde{f} / d \tau)$ means the derivative of $\tilde{f}\left(x^{i}, p^{j}\right)$ along a phase orbit. Since[6]

$$
S_{; j}^{i j}=\int_{\tilde{P}} p^{i} \tilde{L}(\tilde{f}) \tilde{\pi},
$$

we get from (18) and (19) the conservation law $S_{; j}^{i j}=0$. Similarly the particle four-current density, defined by

$$
\mathcal{N}^{i}=\int_{\tilde{P}(x)} \tilde{f} p^{i} \tilde{\pi},
$$

verifies

$$
\mathcal{N}_{; i}^{i}=\int_{\tilde{P}(x)} \tilde{L}(\tilde{f}) \tilde{\pi} .
$$

A simple way of constructing a solution of the Liouville equation is to consider $\tilde{f}$ as a function of constants of the motion. In our case, due to the cylindrical symmetry of the source, the components $p_{z}$ and $p_{\phi}$ of the canonical momentum are conserved. The component $p_{\phi}$ is the particle angular momentum. Thus, a solution, $\tilde{f}\left(p_{z}, p_{\phi}\right)$, of Liouville equation, for particles with $p_{z}=0$, and counterrotating is given by

$$
\tilde{f}\left(p_{z}, p_{\phi}\right)=k \delta\left(p_{z}\right) \delta\left(p_{\phi}^{2}-J^{2}\right)
$$


Here $J$ is the modulus of angular momentum per unit mass. We have the same number of particles with $p_{\phi}=J$ and $p_{\phi}=-J ; k$ is a constant related to the density of particles at a given point on $\Sigma$.

The expression (17) can be written in the form:

$$
S^{i j}=\int_{\tilde{P}(x)} p^{i} p^{j} e^{-2 \psi_{\Sigma}} \delta\left(p^{z}\right) \frac{k}{2 J}\left[\delta\left(p_{\phi}-J\right)+\delta\left(p_{\phi}+J\right)\right] \frac{\sqrt{-g}}{\left|p_{\tau}\right|} d p^{z} d p^{\phi} .
$$

To compute $S^{i j}$ we have to take into account that $p^{\phi}=g^{\phi \phi} p_{\phi}$. The non-zero components of $S^{i j}$ are:

$$
\begin{aligned}
S^{\phi \phi} & =\frac{k J e^{4 \psi_{\Sigma}}}{E R^{5}} \equiv p g^{\phi \phi}, \\
S^{\tau \tau} & =\frac{k E}{J R} \equiv \eta .
\end{aligned}
$$

The quantities $\eta$ and $p$ are the surface energy density and pressure. The shell's full stress-tensor is

$$
S=\eta u \otimes u+p(g+u \otimes u-v \otimes v)
$$

Here $v=e^{-\psi_{\Sigma}} e_{z}$ is the unit vector in the $z$ direction; $E=-p \cdot u$.

From $p \cdot p=-1$ we have:

$$
\frac{e^{2 \psi_{\Sigma}} J^{2}}{R^{2}}=E^{2}-1 \equiv w^{2}
$$

where $w$ is the modulus of the particle linear momentum per unit mass. Introducing the circumference of the shell $2 \pi \mathcal{R}=2 \pi R e^{-\psi_{\Sigma}}$, we have

$$
w=\frac{e^{\psi_{\Sigma}} J}{R}=\frac{J}{\mathcal{R}} .
$$

Thus

$$
\begin{aligned}
\eta & =\frac{k E}{J R}, \\
p & =\eta\left(\frac{E^{2}-1}{E^{2}}\right)
\end{aligned}
$$

Using (20) and (22) we have

$$
\mathcal{N}=v u
$$


where $v$ is the (surface) number of particles density. Integration of (20) gives

$$
v=\frac{k}{J R} \quad \Rightarrow \quad 2 \pi v R=\frac{2 \pi k}{J} \equiv \lambda
$$

where $\lambda \equiv(d m / d z)$ is a conserved quantity: the shell's total rest mass per unit Killing length $z[2]$. The shell's total rest mass per unit of proper length $\left(d z e^{\psi}=1\right)$ is :

$$
\Lambda \equiv-\int \mathcal{N}^{i} u_{i} R e^{-\psi} d \phi=\lambda e^{-\psi}
$$

From (29) and (32) we have $\eta=v E$. Summing up, the parameters $\mathcal{R}, \Lambda, w, E, p, J$ are invariant by the rescaling (2). On the other hand, $R$ y $\lambda$ are scale dependent. The quantity $\lambda$ and $J$ are conserved quantities during a time evolution of the shell.

\subsection{The shell's equation of motion}

From equations (13]14/15) and (26) the junction conditions (7) become:

The $z z$ component:

$$
\psi_{, n}^{+}-\psi_{, n}^{-}=4 \pi(p-\eta)
$$

The $\phi \phi$ component:

$$
-\frac{X^{+}-X^{-}}{R}+\psi_{, n}^{+}-\psi_{, n}^{-}=4 \pi(p+\eta)
$$

The $\tau \tau$ component:

$$
(\gamma-\psi)_{, n}^{+}-(\gamma-\psi)_{, n}^{-}-\left(A^{+}-A^{-}\right)=4 \pi(\eta+p) .
$$

Here $A \equiv e^{2(\gamma-\psi)}(\dot{R} \dot{X}-\ddot{R} X) ; \psi_{, n} \equiv N(\psi)$ is the normal derivative of the gravitational wave field. Equations (34) and (36) are equivalent to:

$$
\begin{aligned}
& \psi_{, n}^{+}-\psi_{, n}^{-}=-\frac{2 \lambda}{\sqrt{1+w^{2}} R}, \\
& X^{+}-X^{-}=-4 \lambda \sqrt{1+w^{2}} .
\end{aligned}
$$

Using the vacuum field equations (3) and (4), and the junction conditions (37) and (38), equation (36) becomes:

$$
\frac{d^{2} R}{d \tau^{2}}=\dot{R} \dot{\psi_{\Sigma}}-R\left[\left(\dot{\psi}_{\Sigma}\right)^{2}+\left(\psi_{, n}^{-}\right)^{2}\right]+\frac{\psi_{, n}^{-} X^{-}}{1+w^{2}}-\frac{\lambda X^{-}}{R\left(1+w^{2}\right)^{\frac{3}{2}}}+\frac{w^{2} X^{-} X^{+}}{R\left(1+w^{2}\right)} .
$$

Equations (37, 39) are the same as (24.a-b-c) of Apostolato and Thorne[2]. 


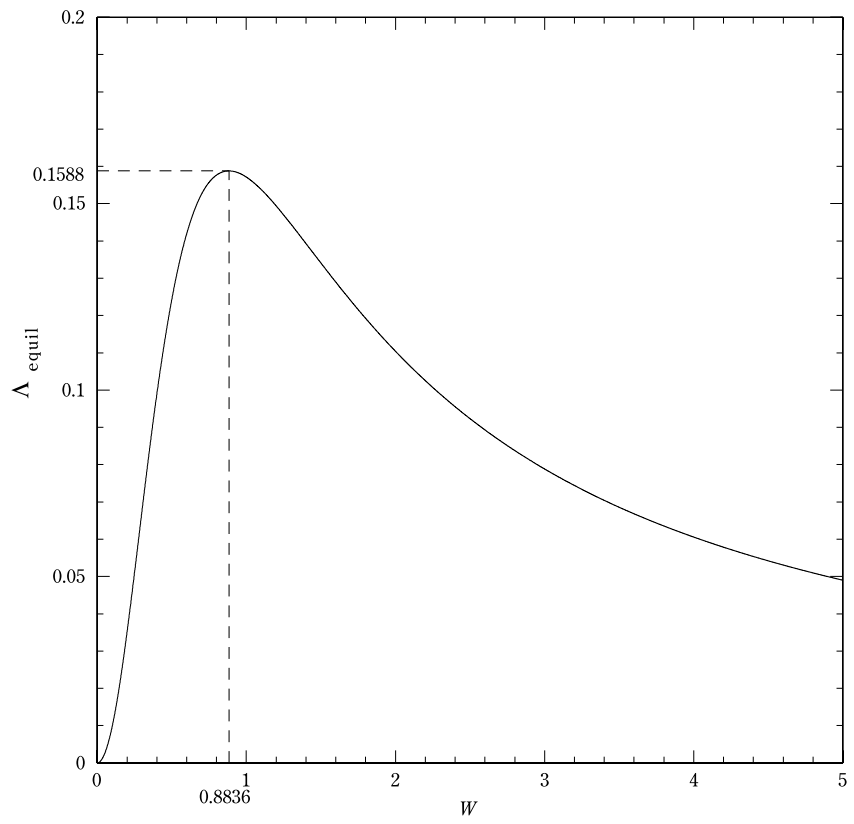

Figure 1: Rest mass per unit proper length for the equilibrium configurations versus the linear momentum per unit rest mass of the particles [equation (46)]. For $w<0.8836$ the equilibrium is stable while for $w>0.8836$ unstable .

\section{Static solutions}

\subsection{The metric}

For a static configuration we have $\dot{R}=0 ; \ddot{R}=0, \psi_{, t}=\psi_{, t t}=0$. Then, in the vacuum outside the shell the field equations (3) and (4) imply

$$
\begin{array}{ll}
\psi^{+}(r)=\psi_{\Sigma}-\kappa \ln (r / R) & r>R, \\
\gamma^{+}(r)=\gamma+\kappa^{2} \ln (r / R) & r>R,
\end{array}
$$

where $\kappa, \psi_{\Sigma}$ and $\gamma$ are constants. Similarly, in the vacuum inside the shell the field equations (3) and (4) plus the boundary conditions (5) give

$$
\psi^{-}=\psi_{\Sigma} ; \quad \gamma^{-}=0 \quad r<R .
$$

From (11) we have

$$
X^{+}=e^{\psi_{\Sigma}-\gamma} ; \quad X^{-}=e^{\psi_{\Sigma}} .
$$

Then, from (38) and (43) we obtain

$$
e^{-\gamma}=1-4 \Lambda \sqrt{1+w^{2}} .
$$


Using that $\dot{R}=0, \dot{\psi}_{\Sigma}=0, \psi_{, n}^{-}=0$ and (44), the equation of motion (39) takes the form:

$$
\ddot{\mathcal{R}} \equiv e^{-\psi} \ddot{R}=\frac{\left(2 w^{2}+1\right)^{2}}{\mathcal{R}\left(1+w^{2}\right)^{3 / 2}}\left[\Lambda_{e q}-\Lambda\right],
$$

where

$$
\Lambda_{\text {equil }}(w)=\frac{w^{2} \sqrt{1+w^{2}}}{\left(2 w^{2}+1\right)^{2}} .
$$

In the case of an equilibrium configuration of the shell, we have:

$$
\ddot{R}=0 \quad \Leftrightarrow \quad \Lambda=\Lambda_{\text {equil }}(w) .
$$

In figure (1) we plot $\Lambda_{\text {equil }}(w)$; the maximum value $\Lambda_{\text {equil,max }}=0.1588$ is reached for $w=0.8836$. Given a constant $\Lambda<0.1588$ we find from (46) two values of $w$ that correspond to equilibrium configurations; or, we may use this equation to obtain a value of $\Lambda$ from $w$. Recall that $w=J / \mathcal{R}$; i.e., for fix $J, \mathcal{R}$ decreases while $w$ increases. For a fix value of $w$ (or $\Lambda$ ), $\mathcal{R}$ is proportional to $J$. Thus, we may have $\mathcal{R}$ approaching zero by just taking $J$ very small.

From the junction condition (37) and equations (40,44), (46) and (47), we obtain

$$
\begin{aligned}
& \kappa=2 w^{2} \\
& \gamma=\ln \left(1+2 w^{2}\right)^{2} .
\end{aligned}
$$

Thus, all the scale invariant quantities that define the static configuration are determined; the scale dependent constant $\psi_{\Sigma}$ may be computed from the relations $e^{-\psi_{\Sigma}}=\Lambda / \lambda=\mathcal{R} / R$, if a value of $\lambda$ or $R$ is given (fixing the coordinate system). In the next subsection we analyze the stability of the equilibrium configurations.

\subsection{Stability analysis}

To determine the nature of the static configurations described in the previous subsection we perform a virtual displacement $\delta \mathcal{R}$ from its equilibrium value. Then, we compute from (45) the virtual change in the acceleration $\ddot{\mathcal{R}}$, around $\ddot{\mathcal{R}}=0$ in the form

$$
\delta \ddot{\mathcal{R}}=\left.\frac{\partial \ddot{\mathcal{R}}}{\partial \mathcal{R}}\right|_{\mathcal{R}_{e q}} \delta \mathcal{R}
$$




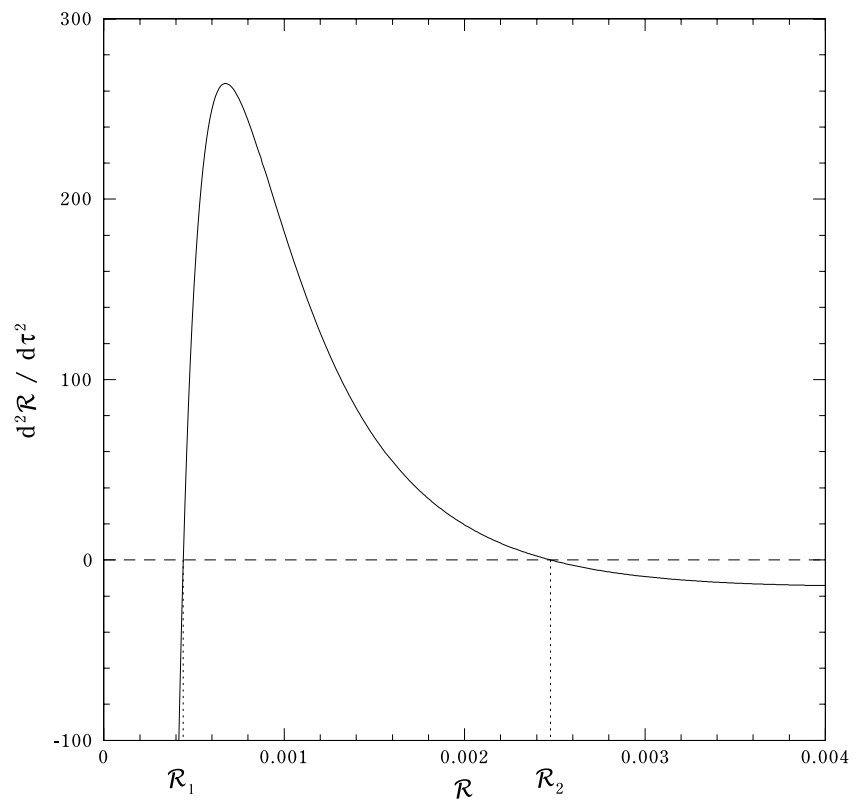

Figure 2: Acceleration vs proper radius of the shell for a momentarily static and radiation free shell[2] given by [45]. The shell with radius $\mathcal{R}_{1}=0.00044$ is unstable while $\mathcal{R}_{2}=$ 0.0024 corresponds to a static stable shell.

The shell is in a static stable configuration if $\left.(\partial \ddot{\mathcal{R}} / \partial \mathcal{R})\right|_{\mathcal{R}_{e q}}<0$; similarly the equilibrium configuration is unstable if $\left.(\partial \ddot{\mathcal{R}} / \partial \mathcal{R})\right|_{\mathcal{R}_{e q}}>0$. From (45), we have

$$
\left.\frac{\partial \ddot{\mathcal{R}}}{\partial \mathcal{R}}\right|_{\mathcal{R}_{e q}}=-\left(\begin{array}{l}
\text { positive } \\
\text { quantity }
\end{array}\right) \times \frac{d \Lambda_{\text {equil }}}{d w} .
$$

From figure (1) we immediately conclude that the stable static configurations correspond to $w<0.8836$; i.e., for values of $\mathcal{R}>J / 0.8836$ (this includes the Newtonian limit); while for $w>0.8836, \mathcal{R}<J / 0.8836$, the equilibrium configurations are unstable. In figure (2) we plot $\ddot{\mathcal{R}}$ as a function of $\mathcal{R}$, for $J=0.001 ; \Lambda=0.1$. Notice that at $\mathcal{R}_{1}=0.00044,\left.(\partial \ddot{\mathcal{R}} / \partial \mathcal{R})\right|_{\mathcal{R}_{1}}>0$ it is sensible larger than $|\partial \ddot{\mathcal{R}} / \partial \mathcal{R}|_{\mathcal{R}_{2}}$ for $\mathcal{R}_{2}=0.0024 ; \mathcal{R}_{1}$ and $\mathcal{R}_{2}$ are the unstable and stable radius, respectively, of the shell. 


\section{Dynamics of two cylindrical shells of counter-rotating particles}

\subsection{The model}

We consider two concentric hollow cylinders composed of counter-rotating particles which define singular hypersurfaces $\Sigma$ and $\Sigma^{\prime}$ of coordinate radius $R$ and $R^{\prime}$, respectively $\left(R^{\prime}>R\right)$, such that the spacetime $M=M^{0} \cup \Sigma \cup M^{-} \cup \Sigma^{\prime} \cup M^{+}$. In the vacuum interior $\left(M^{0}\right)$, intermediate region $\left(M^{-}\right)$, and exterior $\left(M^{+}\right)$, we use canonical cylindrical coordinates $(t, r, z, \phi)$ as before (see (1)). The hypersurface $\Sigma$ is considered in a static, stable equilibrium ( $R=$ const). Therefore the region $M^{0}$ and $M^{-}$are related through $\Sigma$ in the same way as in section 3 . In general, the radius of the singular hypersurface $\Sigma^{\prime}$ will be time dependent. Thus, the metric coefficients in the different regions are given by:

a) $0<r<R$ :

$$
\psi_{0}=\psi_{\Sigma}=\text { constant } ; \gamma_{0}=0 \text {. }
$$

b) $R<r<R^{\prime}(\tau)$ :

$$
\begin{aligned}
& \psi^{-}(r)=-\kappa \ln (r / R)+\psi_{\Sigma} ; \\
& \gamma^{-}(r)=\kappa^{2} \ln (r / R)+\gamma \\
& \kappa=2 w^{2} ; \gamma=\ln \left(1+2 w^{2}\right)^{2} .
\end{aligned}
$$

c) $R^{\prime}(\tau)<r:$

$$
\psi^{+}\left(r, t^{+}\right) ; \gamma^{+}\left(r, t^{+}\right) \quad \text { (wave solutions of the field equations) }
$$

The matching conditions at $r_{\Sigma^{\prime}}=R^{\prime}(\tau)$ require

$$
\begin{aligned}
& \psi^{+}\left(R^{\prime}(\tau), t^{+}(\tau)\right)=\psi^{-}\left(R^{\prime}(\tau)\right) \equiv \psi_{\Sigma^{\prime}}(\tau) \\
& \psi_{\Sigma^{\prime}}(\tau)=-\kappa \ln \left(R^{\prime}(\tau) / R\right)+\psi_{\Sigma} .
\end{aligned}
$$

The shells' surface stress-energy tensors are (see subsection 2.2)

$$
\begin{aligned}
S & =\eta u \otimes u+p(g+u \otimes u-v \otimes v) \\
S^{\prime} & =\eta^{\prime} u^{\prime} \otimes u^{\prime}+p^{\prime}\left(g^{\prime}+u^{\prime} \otimes u^{\prime}-v^{\prime} \otimes v^{\prime}\right),
\end{aligned}
$$

with obvious meanings for the symbols. 


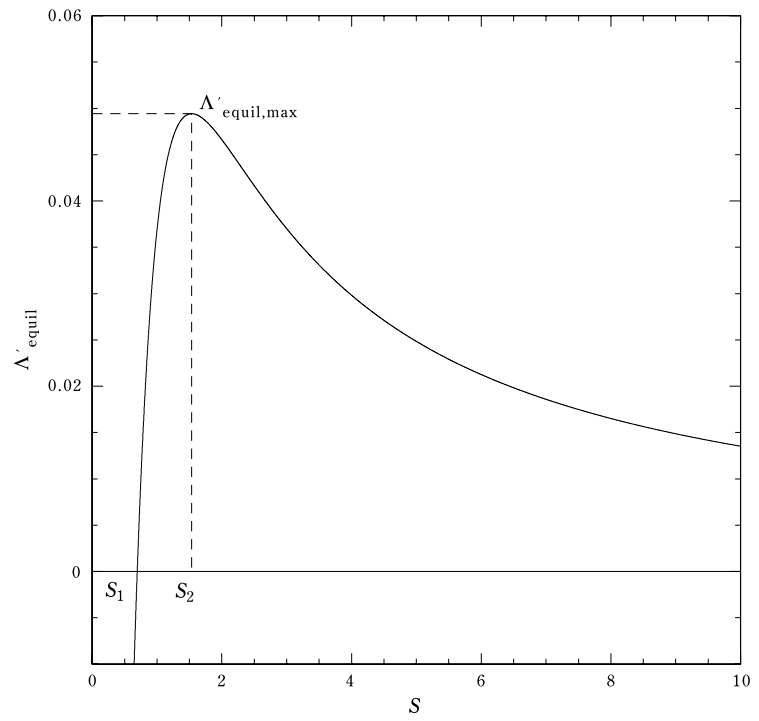

Figure 3: Rest mass per unit proper length of equilibrium configurations of the external shell versus linear momentum per unit rest mass of the particles [equation (61)]. The stable configurations correspond to $s_{1}<s<s_{2}$. For $s>s_{2}$ the equilibrium is unstable; $s_{1}=0.6957, s_{2}=1.5346$.

\subsection{Static solutions}

A static solution corresponds to $R^{\prime}=$ const. The metric coefficients in $M^{+}$are

$$
\begin{aligned}
& \psi^{+}(r)=-\kappa^{\prime} \ln \left(r / R^{\prime}\right)+\psi_{\Sigma^{\prime}}, \\
& \gamma^{+}(r)=-\kappa^{\prime 2} \ln \left(r / R^{\prime}\right)+\gamma^{\prime \prime},
\end{aligned}
$$

where $\kappa^{\prime}, \psi_{\Sigma^{\prime}}, \gamma^{\prime \prime}$ are constants to be determined. Equations (38) and (39) $\left(\dot{R}^{\prime}=\right.$ $0=\ddot{R}^{\prime}$ ) become

$$
\begin{gathered}
\left.\left(e^{-\gamma^{+}}-e^{-\gamma^{-}}\right)\right|_{R^{\prime}}=-4 \Lambda^{\prime} E^{\prime} \\
\Lambda^{\prime}=\frac{\sqrt{1+s^{2}}\left[s^{2}-2 w^{2}\left(1+s^{2}\right)\right]}{\left(1+2 s^{2}\right)^{2}\left(1+2 w^{2}\right)}\left(\frac{\mathcal{R}}{\mathcal{R}^{\prime}}\right)^{\frac{4 w^{4}}{1+2 w^{2}}} \equiv \Lambda_{\text {equil }}^{\prime}\left(s, w, R, J^{\prime}\right) .
\end{gathered}
$$

Where

$$
E^{\prime}=\sqrt{1+s^{2}}, \quad s=\frac{J^{\prime}}{\mathcal{R}^{\prime}}, \quad \gamma^{+}\left(R^{\prime}\right)=\gamma^{\prime \prime}, \gamma^{-}\left(R^{\prime}\right)=\gamma+\ln \left(R^{\prime} / R\right)^{\kappa^{2}} \equiv \gamma^{\prime},
$$




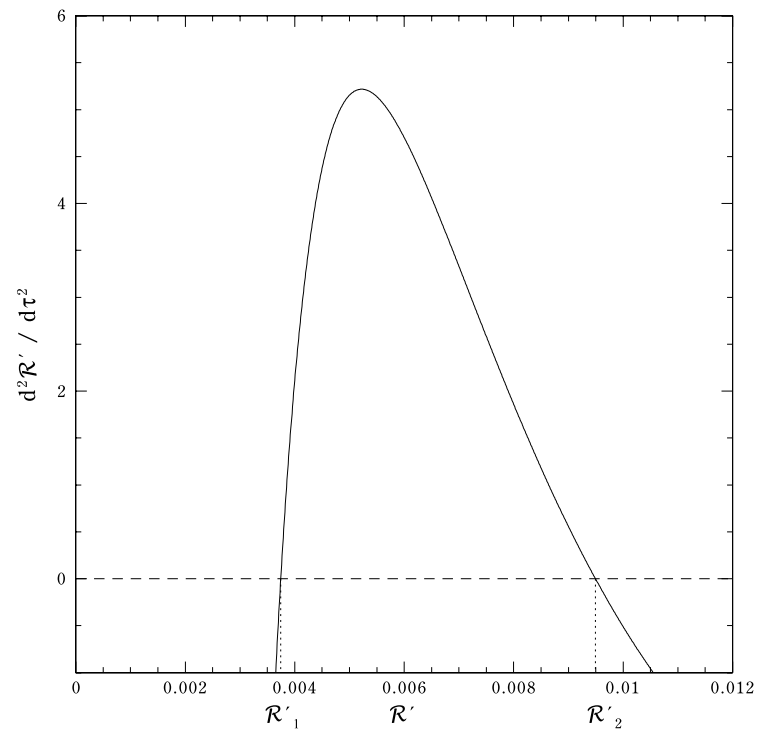

Figure 4: Acceleration vs proper radius of a momentarily static and radiation free external shell[2]. The plot correspond to equation (64). The shell with radius $\mathcal{R}^{\prime}{ }_{1}=0.003744$ is unstable while $\mathcal{R}^{\prime}{ }_{2}=0.009493$ corresponds to a static stable shell.

$J^{\prime}$ is the angular momentum per unit mass of the particles constituting $\Sigma^{\prime}, \mathcal{R}^{\prime}=$ $R^{\prime} e^{-\psi_{\Sigma^{\prime}}}$. Notice that (61) reduces to (46) for $w=0$. From (55) we have

$$
\frac{\mathcal{R}^{\prime}}{\mathcal{R}}=\left(\frac{R^{\prime}}{R}\right)^{K+1} \text {. }
$$

In figure (3) we show $\Lambda_{\text {equil }}^{\prime}\left(s, w, R, J^{\prime}\right)$ as a function of $s$ for $w=0.4038, R=1$ (the values corresponding to the stable static shell of section 3) and $J^{\prime}=0.01$. Considering $\dot{R}=0, \dot{\psi}_{\Sigma^{\prime}}=0,\left.\psi_{, n}^{-}\right|_{R^{\prime}}=-\left(2 w^{2} / \mathcal{R}^{\prime}\right) e^{-\gamma^{\prime}}$, a similar calculation as the one done for the interior shell $(\Sigma)$, shows that (39) becomes

$$
\ddot{\mathcal{R}}^{\prime}=\left(\frac{\mathcal{R}}{\mathcal{R}^{\prime}}\right)^{\frac{4 w^{4}}{1+2 w^{2}}} \frac{\left(1+2 s^{2}\right)^{2}}{\mathcal{R}^{\prime}\left(1+2 w^{2}\right)^{2}\left(1+s^{2}\right)^{3 / 2}}\left[\Lambda_{\text {equil }}^{\prime}-\Lambda^{\prime}\right] .
$$

Thus, the static stable configuration of the exterior shell $\left(\Sigma^{\prime}\right)$ correspond to those values of $s$, such that $\left(\partial \Lambda_{\text {equil }}^{\prime} / \partial s\right)>0$. For $w=0.4038, \mathcal{R}=0.002476, J^{\prime}=0.01$ we have a stable configuration for $0.6957<s<1.5346$ [see figures (3) and 4); $s=$ $1.05335]$.

To complete the static solution we need the expressions for $\kappa^{\prime}$ and $\gamma^{\prime \prime}$. A 

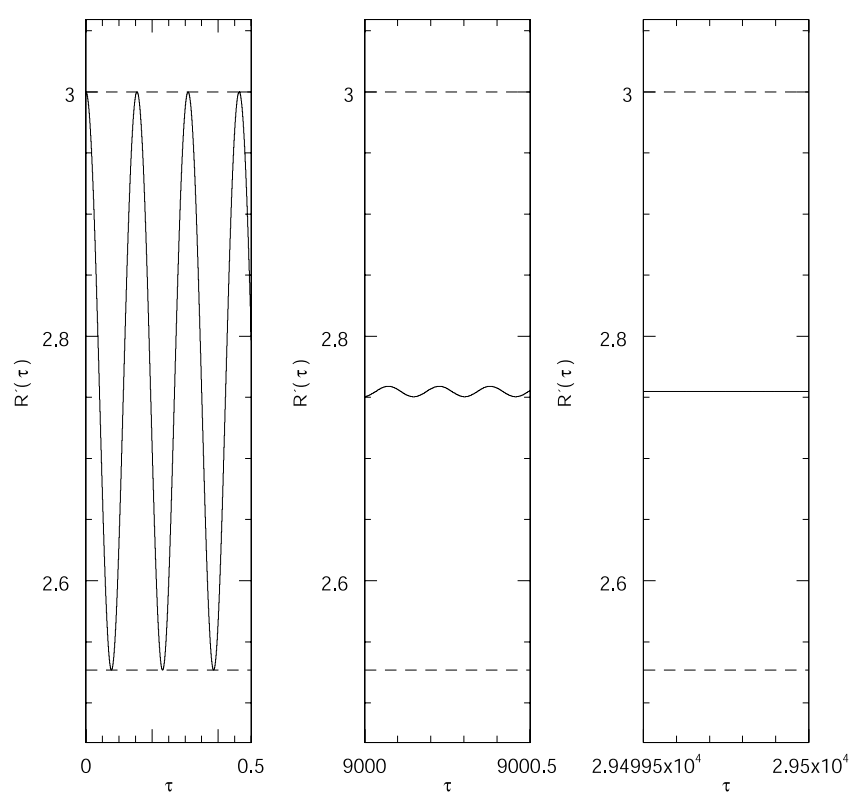

Figure 5: Sequence $R^{\prime}(\tau)$ of a damped oscillating solution for $\lambda^{\prime}=11.6070, \quad J^{\prime}=$ $0.01, R_{i}^{\prime}=3$.

lengthy but straight forward calculation gives:

$$
\begin{aligned}
\kappa^{\prime} & =\frac{2 s^{2}+2 w^{2}\left(1+s^{2}\right)\left(4 s^{2}-2 w^{2}\right)}{1+8 w^{2}\left(1+s^{2}\right)+8 w^{2}\left(1+s^{2}\right)^{2}}, \\
e^{-\gamma^{\prime \prime}} & =\left(\frac{\mathcal{R}}{\mathcal{R}^{\prime}}\right)^{\frac{4 w^{4}}{1+2 w^{2}}} \frac{1+8 w^{2}\left(1+s^{2}\right)\left[s^{2}\left(1+2 w^{2}\right)+2 w^{2}\right]}{\left(1+2 w^{2}\right)^{2}\left(1+2 s^{2}\right)^{2}} .
\end{aligned}
$$

\subsection{Damped oscillating solutions}

Let us now consider a simple time dependent model by assuming that the external shell is time dependent: $r_{\Sigma^{\prime}}=R^{\prime}(\tau)$. We can integrate the equation of motion (39) for $\mathcal{R}^{\prime}(\tau)$ without knowing explicitly the metric for $r>R^{\prime}(\tau)$. Outside the external shell we have a wave solution of the field equations (3, 4) that satisfies the boundary conditions

$$
\begin{gathered}
\psi^{+}\left(R^{\prime}(\tau), t^{+}(\tau)\right)=\psi_{\Sigma^{\prime}}(\tau), \\
\gamma^{+}\left(R^{\prime}(\tau), t^{+}(\tau)\right)=\gamma^{\prime \prime}(\tau),
\end{gathered}
$$

where $\psi_{\Sigma^{\prime}}(\tau)$ is given by (55); $\gamma^{\prime \prime}(\tau)$ may be obtained from the matching condition (38) at the hypersurface $\Sigma^{\prime}$. We specify a particular system choosing an inter- 

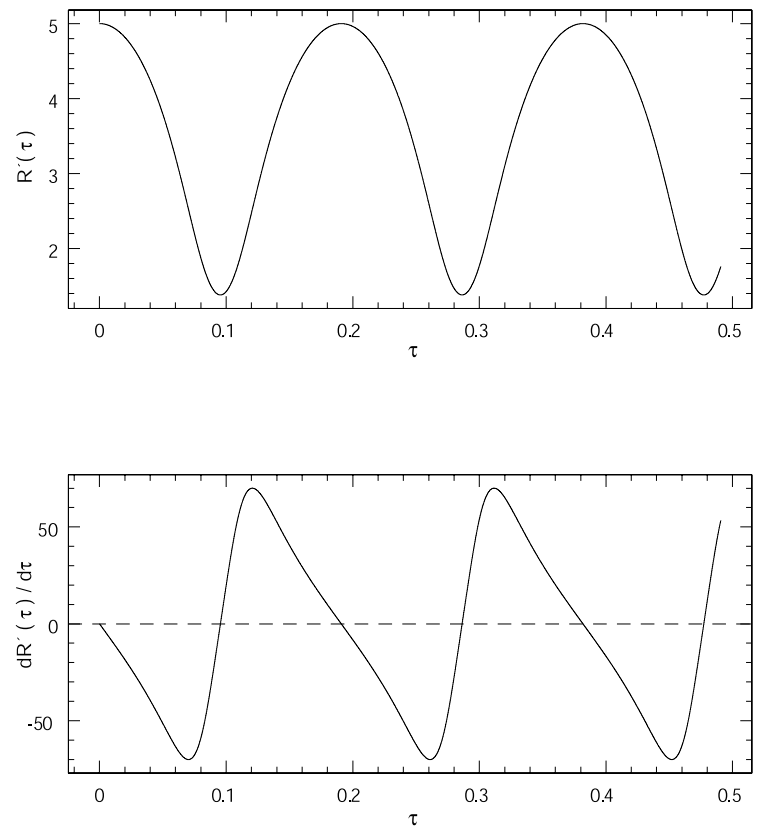

Figure 6: The first oscillations of a shell with $R_{i}^{\prime}=5, \lambda^{\prime}=11.6070, J^{\prime}=0.01$. It shows the asymmetry of the oscillation when it is quite apart from equilibrium.

nal static stable shell, values of the constants $\lambda^{\prime}$ and $J^{\prime}$ and the initial conditions $R^{\prime}(0)=R_{i}^{\prime}, \dot{R}^{\prime}(0)=0$. From (55) we see that the condition $\left(\mathcal{R}^{\prime}(\tau) / \mathcal{R}\right)>1$ is satisfied if $R<R^{\prime}(\tau), \forall \tau$. In figure(5) we show a sequence $R^{\prime}(\tau)$ of a damped oscillating solution, obtained through a numerical integration of (39) for $\lambda^{\prime}=11.6070, J^{\prime}=$ $0.01, R_{i}^{\prime}=3$. The internal solution corresponds to the stable static solution of section $3(\Lambda=0.1, \mathcal{R}=0.002476, J=0.001, R=1)$. We see that the shell oscillates until it reaches the equilibrium radius $R_{e q}^{\prime}=2.7547$. This radius corresponds to the stable configuration with $\Lambda^{\prime}=\Lambda_{\text {equil }}^{\prime}\left(s\left(\tau_{f}\right), w, R, J^{\prime}\right)$, where $\tau_{f} \geq 3 \times 10^{4}$, $s\left(\tau_{f}\right)=1.05335<1.53463$. The same equilibrium configuration is reached for different values of $R_{i}^{\prime}$ in a neighborhood of $R_{e q}^{\prime}$ (we tried $R_{i}^{\prime}=5$, and 2).

\subsubsection{Generalized Komar energy}

All the knowledge that we have of the external solution is reduced to $\Sigma^{\prime}$ as the boundary of $\mathrm{M}^{+}$; i.e, the matter of the external shell. We may calculate a (time dependent) Komar mass[7] per unit of proper length in $\tilde{M}^{+}=M^{+} \cup \Sigma^{\prime}$, using the 
definition

$$
M_{G}=\int_{\bar{\Sigma}} \xi^{b}\left(S_{b}^{\prime a}-\frac{1}{2} S^{\prime} \delta_{b}^{a}\right) \delta(n) d \bar{\Sigma}_{a}
$$

where $\xi^{b}=k \delta_{t^{+}}^{b} ; \bar{\Sigma}: t^{+}=$const $; \delta(n)=\frac{1}{\sqrt{g^{r r}}} \delta(r-R(\tau)) ; d \bar{\Sigma}_{a}=\delta_{a}^{t} \sqrt{-g} d r d \phi d z ;$ $\sqrt{-g}=\sqrt{g^{r r}} r e^{\left(\gamma^{+}-\psi^{+}\right)} ; k$ is a constant. Then (69) becomes

$$
M_{G}=k \int_{0}^{2 \pi} \int_{0}^{e^{-\psi_{\Sigma^{\prime}}}} R^{\prime}(\tau)\left(S_{t}^{\prime t}-\frac{1}{2} S^{\prime}\right) e^{\left(\gamma^{+}-\psi_{\Sigma^{\prime}}\right)} d z d \phi
$$

Using $S^{\prime t t}=\eta^{\prime}\left(X^{+}\right)^{2}+p^{\prime}\left(h^{+}\right)^{t t} ;\left(h^{+}\right)^{t t}=\left(g^{+}\right)^{t t}+\left(X^{+}\right)^{2}$, we have

$$
S_{t}^{\prime t}-\frac{1}{2} S^{\prime}=-\left(\eta^{\prime}+p^{\prime}\right)\left(\frac{1}{2}+\dot{R}^{\prime 2} e^{2\left(\gamma^{\prime}-\psi_{\Sigma^{\prime}}\right)}\right)
$$

choosing $k=-1$, (70) becomes:

$$
M_{G}=\Lambda^{\prime} e^{\gamma^{\prime}} e^{-\psi_{\Sigma^{\prime}}}\left(\frac{1+2 s^{2}}{\sqrt{1+s^{2}}}\right)\left(\frac{1}{2}+\dot{R}^{\prime 2} e^{2\left(\gamma^{\prime}-\psi_{\Sigma^{\prime}}\right)}\right)
$$

to obtain $\gamma^{\prime}$ we have to use the matching condition

$$
X^{+}-X^{-}=-4 \lambda^{\prime} \sqrt{1+s^{2}}
$$

In figure(6) we show $R^{\prime}(\tau)$ and $\dot{R}^{\prime}$ for $\tau<0.5, R_{i}^{\prime}=5$, and $\lambda^{\prime}$ and $J^{\prime}$ equal to the values corresponding to those of figure (5). It is apparent that in the first instances of the motion $\dot{R}^{\prime}$ grows faster than it decreases. It may be inferred, according to order of magnitude estimates using the reduced quadrupole moment of the shell per unit of proper length $[8]$, that the gravitational luminosity is larger after the shell bounces when it reaches its minimum radius [9]. It is clear from (72) that most of the energy radiated away, during the radial oscillation of the external shell, comes from the term proportional to $\dot{R}^{\prime 2}$;i.e, from its kinetic energy as it would be expected.

\subsection{Collapsing and initially expanding solutions}

An interesting set of solutions of equation (39) corresponds to those with $R_{i}$ close to an unstable equilibrium configuration $\left(R_{u e q}^{\prime}\right)$. For instance, if $R_{i}^{\prime}<R_{\text {ueq }}^{\prime}$, the solution of (39) is a collapsing shell that hits the internal shell in a time of order $\tau_{c} \sim 10^{-2}$ [see figure (7)]. On the other hand for $R_{i}^{\prime}>R_{u e q}^{\prime}$ the solution is initially expanding, to settle in a damped oscillating solution with a larger stable radius. An example is shown in figure (8); the unstable equilibrium radius is $R_{\text {ueq }}^{\prime}=1.3660$. The shell settles in a stable equilibrium radius $R_{f}^{\prime} \approx 2.3$. 


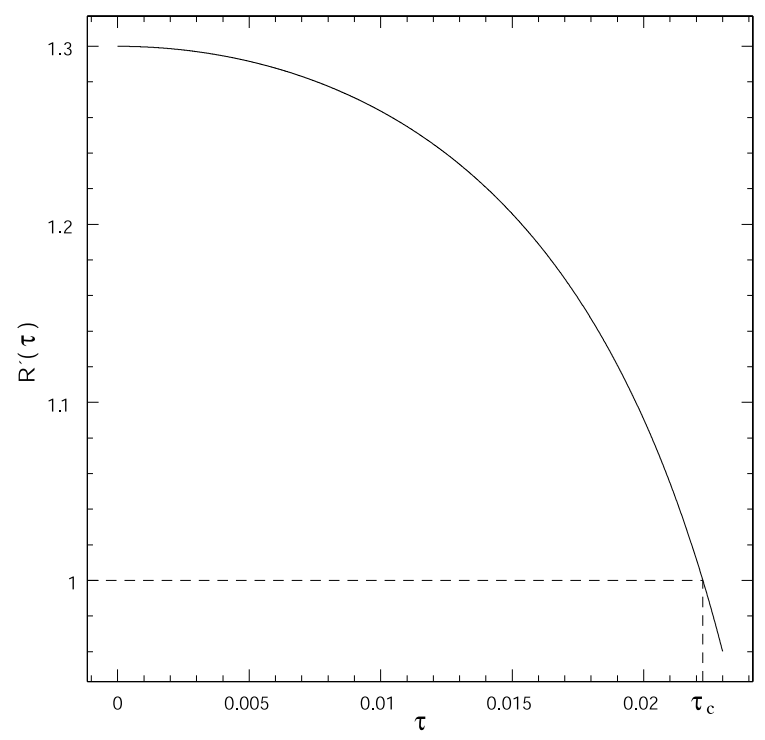

Figure 7: A collapsing solution corresponding to $R_{i}^{\prime}=1.3$ and $R_{i e q}^{\prime}=1.3660 ; \tau_{c}=0.0222$. The constant of the model are $w=0.4038, R=1, J=0.001, J^{\prime}=0.01, \lambda^{\prime}=14.59$.

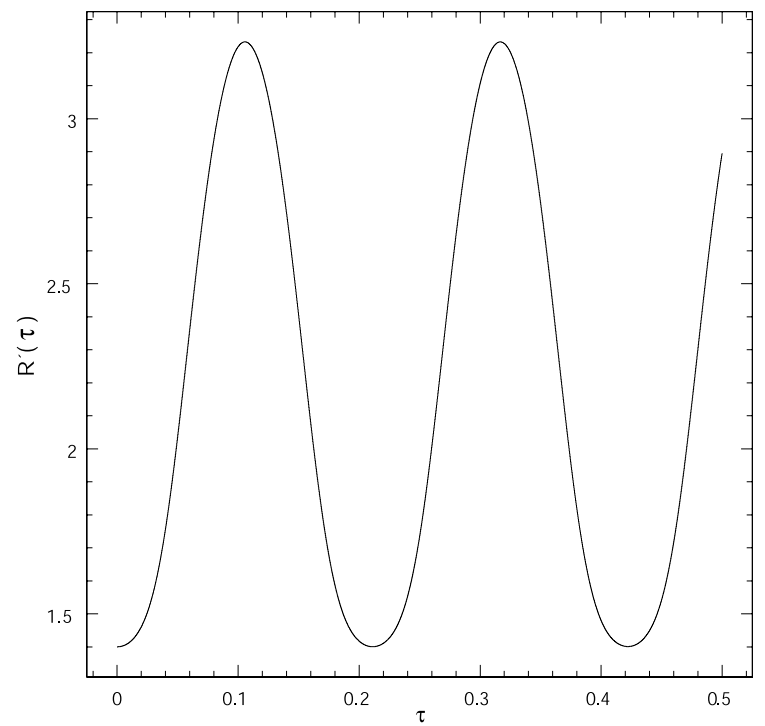

Figure 8: An initially expanding solution. The parameters are the same as in figure(7), $R_{i}^{\prime}=1.4$. 


\section{Final comments}

We have been interested in the relativistic dynamics of cylindrical shells of counter rotating particles. This system have been considered before, in some detail, by Apostolatos and Thorne[2]. Our main contribution to the knowledge of the model is the following:

- We have described the matter composing the shells using the relativistic kinetic theory adapted to a singular hypersurface. This approach has the advantage that the surface energy momentum tensor satisfies the conservation laws if the distribution function is a solution of the Liouville's equation.

- We have analyzed the stability of the equilibrium solutions. We found that the increasing branch of the plot of equation (46) represents the stable equilibrium, while the decreasing part of the plot are the unstable equilibrium configurations. This figure was first introduced by Apostolato and Thorne[2] for another purpose.

- The study of the equilibrium solutions was extended to two concentric shells. The results are similar as for only one shell [Compare figures (1, 2) with (3, 4)].

- Armed with the previous results, we analyzed the damped oscillating solutions of the external shell in the two shells model. These solutions converge to a stable static configuration, depending on the values of the constant of the motion and initial conditions.

- The most interesting result was to show that the solutions that start with a radius smaller than the corresponding radius of a unstable static solution, collapses to hit the internal shell, whose proper radius may be quite small, depending on the angular momentum per unit mass of the particles of the internal shell. The spacetime that results is free of singularities as long as this angular momentum is not null.

Finally, we think that more information can be obtained from the equation of motion of a time dependent shell, about the basic qualitative features of generation of gravitational waves and the behavior of the matter conforming such shells, in the limit of very small radius. An interesting problem would be to relate the motion of the shell with the C-energy[2, 3] carried away by gravitational waves, while in the interior of the shell we have a superposition of ingoing and outgoing 
cylindrical gravitational waves that satisfies the boundary conditions (5). This and related topics may be discussed elsewhere.

\section{Acknowledgements}

The authors VHH and DEB are very grateful to CONICET of Argentina, and SECyT of the Universidad Nacional de Córdoba for financial support.

\section{References}

[1] For instance: M. Sharif and Z. Ahmad, Gen. Rel. Grav. 39, 1311 (2007); K. Nakao, Y. Kurita, Y. Morisawa, T. Harada, Prog. Theor. Phys. 117, 75 (2007); K. Nakao and Y. Morisawa, Phys. Rev. D71, 124007 (2005); L. Herrera and N. Santos, Class. Quant. Grav. 22, 2407 (2005); S. Masafumi, Phys. Rev. D69, 124030 (2004), S. Goncalves and S. Jhingan, Int. J. Mod. Phys. D11, 1469 (2002).

[2] T.A. Apostolatos and K.S. Thorne, Phys. Rev. D46, 2435 (1992).

[3] K.S. Thorne, Ph.D. thesis, Princeton University, 1965; available from University Microfilms Inc., Ann Arbor, Michigan.

[4] A. Einstein and N. Rosen, J. Franklin Inst. 223, 43 (1937).

[5] W. Israel, Nuovo Cimento 44B, 1 (1966); 48B, 463 (1967).

[6] J. Ehlers, "General Relativity and Kinetic Theory", in General Relativity and Cosmology, course 47. Proceedings of the International School of Physics "Enrico Fermi", edited by R.K. Sachs (Academic Press, New york, 1971) p. 1 .

[7] A. Komar, Phys. Rev. 113, 394 (1959). See also: J. Winicour, "Angular Momentum in General Relativity" in General Relativity and gravitation, Edited by A. Held, V2, p. 74 (Plenum Press New York, 1980).

[8] C. W. Misner, K. S. Thorne and J. A. Wheeler, Gravitation (Freeman, San Francisco, 1973) Chapter 36, §36.2.

[9] Compare with T. Piran, Phys. Rev. Lett. 41, 1085 (1978). 